\title{
Dramaturgie de la « folie » dans le théâtre jeune public
}

Madness Dramaturgies in the Theatre for Young People

Johanna Biehler

\section{CpenEdition}

\section{Journals}

Édition électronique

URL : https://journals.openedition.org/recherchestravaux/789

DOI : 10.4000/recherchestravaux.789

ISSN : 1969-6434

Éditeur

UGA Éditions/Université Grenoble Alpes

Édition imprimée

Date de publication : 1 décembre 2015

Pagination : 117-126

ISBN : 978-2-84310-314-8

ISSN : 0151-1874

Référence électronique

Johanna Biehler, «Dramaturgie de la « folie » dans le théâtre jeune public », Recherches \& Travaux [En ligne], 87 | 2015, mis en ligne le 01 janvier 2017, consulté le 29 octobre 2021. URL : http://

journals.openedition.org/recherchestravaux/789 ; DOI : https://doi.org/10.4000/recherchestravaux. 789 
Johanna BIEHLER

Université de Pau et des Pays de l'Adour

\section{Dramaturgie de la «folie» dans le théâtre jeune public}

Si la maladie mentale est un sujet récurrent au théâtre, et cela pour un lectorat adulte, il peut sembler légitime de se poser la question de sa présence dans les écritures dramatiques dites pour les jeunes. Les auteurs considèrent qu'il faut absolument parler aux enfants des «sujets difficiles», selon l'expression de Joël Pommerat ${ }^{\mathrm{T}}$, mais que cela demande des dramaturgies adaptées dont nous étudierons quelques exemples.

La folie et ses excès sont présents au théâtre depuis ses origines et nous ont offert des œuvres dramatiques considérées comme des références aujourd'hui : Ajax de Sophocle ou Le Roi Lear de Shakespeare. La disparition de la folie des nosographies médicales n'a en rien freiné l'attrait des auteurs pour les mystères de la psyché humaine. Le théâtre contemporain a intégré, d'un point de vue esthétique et dramaturgique, les progrès de la médecine en ce qui concerne la maladie mentale et offre des pièces se déroulant dans des hôpitaux psychiatriques, mettant en jeu le désespoir des personnages face au diagnostic, l'errance meurtrière du «fou » en liberté... Et le théâtre pour les jeunes ne fait pas exception.

Si les troubles du comportement ne sont pas considérés habituellement comme un sujet adapté aux enfants, à la frontière entre raison et déraison, les auteurs dramatiques n'hésitent pourtant pas à proposer des textes où la maladie mentale occupe une place centrale. S'ils considèrent qu'il n'y a plus de sujet tabou, cela demande des dramaturgies particulières car il s'agit aussi de ne pas heurter la sensibilité de ce public et lectorat. À travers trois situations mettant en

I. Cité par A. Gablin, Le théâtre jeune public : un espace en débat, mémoire de recherche, Institut d'études politiques de Toulouse, 2007, p. 24. 
scène un personnage enfant face à la maladie mentale, nous verrons comment les auteurs concilient leur désir d'écrire sur la thématique de la maladie mentale et leur engament éthique envers le jeune spectateur ou le jeune lecteur.

\section{Folie de l'enfance ou l'imagination débordante}

La folie a perdu, au fil des siècles, toute signification médicale pour se rapprocher de l'idée d'exubérance ou d'extravagance. Toutefois, la différence entre un petit grain de folie et un véritable trouble du comportement est parfois difficile à établir, et cela est d'autant plus vrai chez un enfant.

La folie du personnage principal de Louise / les ours de Karin Serres commence à se manifester quand elle se met à voir des ours blancs et transparents qui suivent les gens. Son père Ian et sa grande sœur adolescente Élinor ne peuvent imaginer que deux raisons à ces soudaines apparitions : Louise se drogue ou elle est malade. Les deux options ne sont en aucun cas agréables à envisager à propos d'une enfant de onze ans. La construction dramaturgique de la pièce amène le lecteur vers une troisième possibilité que les membres de la famille de Louise n'envisagent pas encore : les ours existent vraiment.

Les personnages abordent cet événement de deux façons diamétralement opposées : Ian accorde le bénéfice du doute à sa fille puisqu'il parle d'un "don » et cela le fait plutôt rêver. Élinor, quant à elle, préfère se moquer de Louise avec des mots comme «tarée», «cinglée», "frappée» et des expressions comme "péter un plomb». Si cela semble faire partie des taquineries habituelles entre sœurs, Élinor est la seule à envisager les conséquences possibles si Louise se révèle être vraiment malade et non plus une petite fille à l'imagination débordante : «Personne la croira, ils vont l'enfermer, la bourrer de médocs, l'électrochoquer, la lobotomiser ${ }^{2}$ !» L'accumulation de références à la maladie mentale peut instaurer un doute dans l'esprit du lecteur ou du spectateur quant à la véritable nature des visions de Louise.

Cet élément dramaturgique semble s'adresser à des adultes; or la pièce est considérée comme un texte pour les jeunes, et cela précisément grâce à la présence des ours car ils rappellent le nounours en peluche rassurant. Les histoires pour enfants regorgent d'animaux magiques et protecteurs qui reposent essentiellement sur l'idée que l'enfant ne serait pas encore perverti par le monde des adultes et garderait une capacité à vivre dans un état plus proche de la nature. Sigmund Freud explique ainsi dans Totem et Tabou cette proximité entre les enfants et les animaux :

2. K. Serres, Louise / les ours, Paris, L'École des loisirs, coll. «Thêâtre», 2006, p. 53. 
L'enfant n'éprouve encore rien de cet orgueil propre à l'adulte civilisé qui trace une ligne de démarcation nette entre lui et tous les autres représentants du règne animal. Il considère sans hésitation l'animal comme son égal; par l'aveu franc et sincère de ses besoins, il se sent plus proche de l'animal que de l'homme adulte qu'il trouve sans doute plus énigmatique ${ }^{3}$.

Lours cristallise le désir de Louise de rester une enfant et de ne pas basculer dans le monde effrayant des adultes. Or, elle arrive à un âge où une telle obstination commence à devenir inquiétante pour son entourage. Où se trouve la frontière entre le pathologique et l'imagination? La famille de Louise pense qu'elle se crée des amis imaginaires avant que son père ne voie, à son tour, ces créatures. Cet effet dramaturgique semble entraîner le lecteur ou le spectateur dans une dimension fantastique, mais qu'en est-il quand ce type de fantasmes commence à témoigner d'une certaine violence, comme dans Les Aventures d'Auren le petit serial killer de Joseph Danan?

Les animaux ont aussi une place importante dans cette pièce, car le héros rêve que son double, l'enfant-sanglier, se laisse aller à suivre ses pulsions les plus instinctives : tuer quiconque ose se mettre en travers de sa route, enlever la petite fille rencontrée sous un hêtre, suivre les voix (d'enfants) qui l'incitent à tuer... le temps.

Ces fantasmes sèment le désordre et Auren se trouve ainsi obligé de fuir, en laissant des cadavres partout où il passe. Sa propre mère va jusqu’à le qualifier de «monstre» à plusieurs reprises, elle avance même que sa dent cariée est «toute noire / Comme son âme^». Bernadette Bost rapproche Auren d'un autre criminel schizophrène à la cavale meurtrière, le personnage éponyme de la pièce de Bernard-Marie Koltès Roberto Zucco : "Auren a beau apparaître comme "le plus gentil petit garçon de la terre", il se rêve tueur, petit frère de Roberto Zucco quand il confie à une gamine, à l'instar du héros de Koltès, son nom secret d'assassin sans raison'.» Les deux pièces sont, de plus, construites selon une dramaturgie proche du «drame à station» médiéval et remis au goût du jour par Strindberg avec le jeu de rêve'.

Dans le tableau intitulé «Des pays étranges et lointains», Auren se retrouve dans une sorte de camp de vacances où il participe, malgré lui, à un nouveau

3. S. Freud, Totem et Tabou, trad. S. Jankélévitch, Paris, Payot, coll. «Petite bibliothèque», I97I, p. I47.

4. J. Danan, Les Aventures d'Auren le petit serial killer, Arles, Actes Sud, coll. «Poche théâtre», 2007, p. 29.

5. B. Bost, «Le rire cruel de Joseph Danan", Recherches et Travaux, n ${ }^{\circ}$ 69, 2006, p. III.

6. Le jeu de rêve cherche à «imiter la forme incohérente, en apparence logique, du rêve. Tout peut arriver, tout est possible et vraisemblable» (A. Strindberg cité par J.-P. Sarrazac, «Jeu de rêve», dans J.-P. Sarrazac [dir.], Lexique du drame moderne et contemporain, Belfort, Circé, coll. «Poche», 2005, p. I07). 
divertissement appelé «jeu du bourreau». Une didascalie décrit cette scène comme étant "comme un rêve", bien que cela se révèle plus proche du cauchemar puisque ce «rêve" s'achève avec l'assassinat des trois oursons. Les personnages meurent puis reviennent plus tard sur scène sous d'autres identités, mi-hommes mi-animales, représentées par des masques. C'est justement le potentiel des possibles accordé par le rêve et le théâtre qui intéresse Joseph Danan. Il explique dans une note liminaire : «C'est peut-être pour ça que je suis devenu écrivain : pour prendre des libertés, et en donner. Pour tuer ceux qui m'embêtent et courir loin de ma mère ${ }^{7}$.» Le rêve permet tout cela et devient un modèle dramaturgique. Personne ne meurt vraiment à cause des agissements d'Auren, les prétendues victimes reviennent toujours pour incarner d'autres personnages et porter de nouveaux masques. Tous les acteurs seront présents pour saluer à la fin de la représentation. Ainsi, la dimension métathéâtrale permet de dénoncer la violence omniprésente en rappelant que tout cela n'est, finalement, qu'un jeu où la mort et la folie sont soumises aux règles du faire semblant.

Le point commun que nous pouvons trouver entre Auren et Louise est l'excuse que leur donne leur jeune âge : avoir une imagination débordante à la préadolescence n'est pas en soi inquiétant. En revanche, que doit-on en penser si la situation perdure? Une personne voit des choses invisibles, parle tout haut pendant des heures à quelqu'un qui n'est pas là... La conclusion à tirer d'une telle situation est qu'il s'agit d'une personne mentalement dérangée, bien qu'elle ne soit pas dangereuse si les symptômes en restent là. Or, le cas d'Auren est encore plus grave : se laisser aller à la violence durant une sorte de "rêve éveillé» n'est pas mauvais et cela peut même être considéré comme très sain, mais que se passera-t-il si un jour cela ne suffit plus à l'enfant rêveur et qu'il passe à l'acte?

Louise et Auren, à leur âge et à ce stade de développement, ne sont pas inquiétants et leur jeunesse, ainsi que les «détournements" (les animaux, les masques, l'humour...), mettent en place une dramaturgie qui permet au texte de ne pas effrayer les jeunes lecteurs ou spectateurs. Ces personnages sont "fous» dans l'acception courante du mot, mais non pathologique. Les auteurs dramatiques contemporains n'hésitent pas à aller plus loin et à mettre en scène dans leurs pièces des enfants souffrant de maladies mentales.

7. J. Danan, Les Aventures d'Auren le petit serial killer, ouvr. cité, p. 83. 


\section{L'amour médecin de l'enfant malade}

Si le cas de l'enfant malade est mis en scène, les auteurs ont recours à une méthodologie dramaturgique particulière qui s'intéresse tout spécialement à la fin du texte avec une remise en question du happy end perçu comme une caractéristique inhérente des textes s'adressant à un jeune lectorat.

La pièce Je m'appelle Non de Liliane Atlan est l'adaptation d'un texte plus ancien de l'auteur, Les Passants ${ }^{8}$, qui évoque les difficultés pour une jeune fille juive à vivre avec le traumatisme des camps de concentration et la culpabilité du survivant. Cette difficulté provoque chez elle une anorexie. Les conséquences de la maladie sont très bien décrites dans la pièce, à la fois en ce qui concerne le personnage principal mais aussi son entourage car l'auteur elle-même a survécu à ce trouble alimentaire. La pièce se joue sur deux lignes temporelles et adopte ainsi une dramaturgie épique avec une alternance de narration et de dialogues : ce sont à la fois les souvenirs racontés de la Vieille Non et son présent qui forment le texte. Elle se présente ainsi :

Dans une maison riche, à Marseille, à la fin d'une guerre, une jeune fille se laisse mourir de faim.

Elle a quatorze ans, ou quinze.

Elle s'appelle Non, de son prénom, Mais Je M’en sortirai, de son nom de famille. PETITE Non apparaît.

VIEILLE Non

Elle va au lycée, elle étudie avec rage, elle fait de longues marches forcenées dans les calanques, mais depuis des mois elle ne mange plus, on voit ses os, elle commence à perdre la mémoires .

L'univers évoqué est foncièrement réaliste dans ses références à la maladie grâce à l'expérience de l'auteur et en particulier dans sa description des difficultés que peut rencontrer une famille à se reconstruire après la Shoah. Liliane Atlan était juive et elle pouvait donc s'inspirer de ses souvenirs et de son entourage. Cette dimension autobiographique est en même temps mise à distance grâce à l'humour présent dans le choix des noms, ce que Marie Bernanoce appelle les «noms motivés», et qui "participe de l'épicisation de l'écriture, favorisant ainsi une mise à distance à l'opposé de tout naturalisme ${ }^{\mathrm{I0}}$ ». L'humour transparaît dans certaines anecdotes racontées par les personnages

8. L. Atlan, Les Passants suivi de Corridor paradise concert brisé, Paris, L'Harmattan, coll. «Écritures», I998.

9. L. Atlan, Je m’appelle Non, Paris, L'École des loisirs, coll. "Théâtre», I998, p. I3.

Io. M. Bernanoce, À la découverte de cent et une pièce, Montreuil-Grenoble, Éditions Théâtrales - SCÉRÉN, 2006, p. 42. 
(JE ME CRÉERAI MOI-MÊME explique en riant que, ayant eu le malheur de dire un jour que les poires prévues pour le dessert étaient bonnes, la grand-mère de Non se fit un devoir d'en proposer à chaque repas et que depuis il n'en a plus mangé une seule) ou encore dans les noms de psychiatres (J'EN FAIS MON AFFAIRE, JE FAIS FUIR LES MECS Ou encore JE ME TAIS ET J'EMPOCHE...) Leur efficacité thérapeutique est tout de suite remise en cause, la psychiatrie n'aura finalement pas grand-chose à voir avec la guérison de NoN, car ce qui la sauvera véritablement, c'est l'amour.

En effet, Non guérit de son anorexie grâce à l'amour d'un personnage appelé Je DÉCOUVRIRA LE SENS DE LA VIE. Cette rencontre lui permet de se tourner enfin vers l'avenir qui semblait bien sombre jusque-là. La rémission de NoN semble respecter la convention du happy end. Pourtant, un autre personnage malade est mentionné à la fin de la pièce. NonetTe, la nièce de Non, souffre du même mal et sa tante semble persuadée de sa guérison future. Or, rien n'est dit à ce propos et plusieurs interprétations sont possibles : la jeune fille peut se rétablir mais elle peut aussi en mourir, Non projette son expérience sur sa nièce. Toutefois, il est plus gai de penser que NonETTE guérira. Il s'agit là d'une convention de la part des auteurs qui écrivent pour la jeunesse : si les fins heureuses ne sont plus perçues comme des éléments incontournables du genre, la dramaturgie amène plusieurs niveaux d'interprétation de la pièce et certains sont plus heureux que d'autres. NonetTe rencontrera peut-être l'amour et cela la guérira, mais le contraire reste envisageable.

C'est une fin analogue que nous trouverons dans Les Petits Mélancoliques ${ }^{\text {II }}$ de Fabrice Melquiot. Le personnage principal, Petit Tom, se décrit comme atteint d'une nouvelle crise de mélancolie, maladie dont il souffre depuis le décès de sa mère. Il est persuadé qu'il va bientôt mourir suite à un rêve qu'il pense être prémonitoire. La fascination pour la mort dont témoigne le personnage principal provient du fait qu'il la perçoit comme la seule solution pour mettre fin à ses souffrances. Il s'agit d'une caractéristique typique du mélancolique qui «n'envisage l'achèvement que sous l'angle de la mort ${ }^{12}$ ».

Le côté très macabre et sinistre de tels propos dans la bouche d'un enfant est modéré par les créatures étranges qui rôdent dans la pénombre autour de Tom (le Marchand de sable, la Tempête...). Elles évoquent une atmosphère onirique tout comme dans Les Aventures d'Auren. La langue poétique des didascalies s'inscrit plutôt dans une esthétique romantique mêlée de surréalisme tandis que les éléments plus sombres nous entraînent dans un univers proche du conte de fée, mais dans la lignée des contes de fées archaïques pleins de

II. F. Melquiot, Les Petits Mélancoliques, Paris, L'École des loisirs, coll. «Théâtre», I999.

I2. M. Guérin, La Pitié, Arles, Actes Sud, coll. «Le Génie du philosophe», 2000, p. IO3. 
violence. Ce mélange étrange de poésie noire et d'histoires enfantines presque mythologiques peut paraitre difficile à affronter pour des jeunes quelles que soient les conditions de réception : l'incarnation sur scène donne l'illusion de la présence de ces créatures devant un public (ce qui peut devenir très effrayant), la lecture est un exercice par essence solitaire où l'enfant est seul avec son imagination. Il faut rappeler que les recherches en psychanalyse sur ce type de récits ont montré que les contes sont nécessaires à la construction de la psyché de l'enfant. Dans ce cas, pourquoi serait-ce insupportable sur une scène de théâtre?

Pour que la pièce ne soit pas trop triste, Fabrice Melquiot recourt à une forme de théâtre dans le théâtre. Tom découvre à travers le jeu qu'il peut faire son deuil et continuer à vivre malgré le décès de sa mère. Ce sujet peut sembler morbide pour une pièce destinée à la jeunesse, mais le texte déjoue lui-même l'illusion théâtrale et rappelle indirectement que tout cela n'est qu'une fiction. L'un des compagnons de Tom, Mite l'ermite, est un ancien acteur qui connait bien le problème que pose la mort au théâtre.

Quand j'étais acteur, j'ai fait semblant de mourir des centaines de fois, dans des centaines de théâtre, et des centaines de foules m’ont regretté. On m’a écrit des lettres pour me dire : pourquoi vous êtes mort? J'ai répondu des centaines de fois; les gars, je fais semblant, je ne suis pas mort ${ }^{13}$.

Après avoir joué (dans tous les sens du terme) une saynète extraite de Romilette et Julio, les personnages sortent de leurs rôles pour analyser la représentation comme le font les acteurs et le metteur en scène. Si Petit Tom prend du plaisir au jeu, et donc à la vie, c'est le signe que sa dépression n'est peut-être pas si grave et que la guérison est envisageable pour lui. C'est le point commun que nous trouvons à ces deux textes, Les Petits Mélancoliques et Je m'appelle Non: les deux héros sont sauvés de la maladie par l'amour et le récit. Pour la jeune fille anorexique, il s'agit de l'amour pour une autre personne mais aussi du fait de raconter son histoire, la parole opérant une catharsis. Petit Tom se découvre une passion pour le théâtre et choisit de se mettre en scène en jouant des saynètes qui évoquent le psychodrame.

La famille est un élément important dans les textes de Liliane Atlan ou dans la seconde pièce à l'étude de Fabrice Melquiot afin de rassurer l'enfant spectateur ou lecteur : le jeune personnage malade n'est pas seul face à la maladie, mais où trouver un allié ou de l'aide quand un proche est malade?

13. F. Melquiot, Les Petits Mélancoliques, ouvr. cité, p. 19. 


\section{Parler de la maladie à un enfant}

Le texte dramatique Blanches de Fabrice Melquiot est un duo qui met en scène une enfant et sa grand-mère. Mémé Blanche n'est pas seulement une vieille dame un peu excentrique, elle souffre de la maladie d'Alzheimer. Sa petitefille, surnommée "Ouais» car elle répond toujours par ce mot, nous rapporte que son père dit de Mémé Blanche «qu'elle est psychopathe». Ce qui peut passer pour un jugement très dur de la part d'un beau-fils n'est en fait que de bonne guerre parce que la vieille dame n'est pas si innocente que cela, toujours selon Ouais : "Mémé dit que c'est normal que Papa dise ça, vu que primo, c'est son gendre, deuzio, il est débile.» Rappelons que de manière figurée et courante, voire familière pour l'adjectif "débile», la débilité désigne la faiblesse mentale. D'après Ouais, tous les adultes de son entourage sont plus ou moins déséquilibrés (elle évoque aussi la dépression de sa maîtresse d'école), et malgré tout elle semble très bien s'en accommoder.

$\mathrm{Au}$ fil du texte, Ouais voit la personnalité de sa grand-mère changer et devenir de plus en plus étrange, voire violente. Mémé Blanche ne se souvient plus de ce qui est advenu il y a seulement quelques instants ni de l'identité des personnes qui se trouvent en sa présence. Elle devient quelqu'un d'autre et Ouais voit disparaître petit à petit sa grand-mère fantasque et adorée. L'enfant a besoin d'explications pour comprendre cela et les informations données par ses parents ne sont pas rassurantes. Ouais ressent alors le besoin de confier ses peurs à son grand-père décédé à travers des lettres :

Ouais. Cher Pépé Lulu,

Mémé est devenue bizarre. Elle fait des choses bizarres, elle en dit aussi. Des fois, j’ai peur de Mémé. Je n’avais jamais eu peur de Mémé. Maman m’a dit qu’elle avait un truc à la tête, un truc avec un nom bizarre. Il parait que Mémé ne sera plus jamais comme avant, quand elle était bizarre mais juste un peu quand même. Papa m’a dit que ce qu'elle avait, ça n’allait pas s'arranger.

[...]

Pépé Lulu, est-ce que plus tard, j'aurai un truc à la tête, moi aussi?

Et mes enfants, ils auront des trucs à la tête ${ }^{\mathrm{T}}$ ?

Quelque chose a changé chez Mémé Blanche et Ouais l'a bien perçu car elle avoue : «Des fois, j'ai peur de Mémé. Je n’avais jamais eu peur de Mémérs.» Il $\mathrm{y}$ a une raison à ce changement de personnalité qui n'a absolument rien à voir avec le fait que Mémé Blanche aurait soudainement cessé d'aimer sa petitefille. Elle est malade et cette maladie a un nom étrange et difficile à retenir.

I4. F. Melquiot, Blanches, Paris, L’Arche, coll. "Théâtre jeunesse», 20IO, p. 43-44.

I5. Ibid., p. 43. 
La pièce aborde ainsi la délicate question que se posent tous les parents confrontés à une telle situation : faut-il parler sans détour de la maladie d'Alzheimer ou se taire pour préserver l'enfant? Les parents de Ouais ont clairement choisi la première option ("Papa m'a dit [...] ce qu'elle avait $\left.{ }^{16} »\right)$ et surtout l'absence de guérison possible. Cette franchise parentale peut sembler très dure de prime abord, mais les psychiatres ou les associations de malades préconisent le dialogue avec l'enfant. Pour Fabrice Melquiot, l'attitude à adopter est très claire : dans un entretien, à la question «Peut-on parler de tout avec les enfants?», il répond : «Oui, il suffit de trouver la manière. Ils peuvent tout recevoir, mais il faut trouver les mots, la forme ${ }^{17}$."

Quelles peuvent être les conséquences, pour Ouais, de la maladie de sa grand-mère? Nous pouvons dire que cet événement l'amène doucement vers le monde des adultes. Elle finit par abandonner son surnom pour adopter son prénom définitif. Il s'agit du prénom déjà porté par sa grand-mère, ce qui nous éclaire sur le mystérieux pluriel du titre de la pièce. L'enfant prend sa place dans une lignée de femmes même s'il est difficile d'entendre les questions que Ouais se pose sur le côté héréditaire de la maladie. L'aspect matrilinéaire est mis en avant par la construction de la pièce et la maladie, chez les Blanches, est une histoire de famille. Comme le prénom et les robes en dentelle, cela se transmet de génération en génération. Toutefois, la maladie fait moins peur quand on l'affronte à plusieurs.

La folie et la maladie mentale sont des thématiques qui peuvent faire peur quand on s'adresse à un lectorat ou un public d'enfants. L'idée reçue veut qu'il faille leur parler de certaines choses et en ignorer d'autres afin de préserver leur innocence de la dureté de la vie. Or, les actions de sensibilisation à l'art, à l'exemple du prix Collidram, montrent que les comités de jeunes lecteurs récompensent les textes abordant des sujets sérieux qui donnent matière à réflexion et les reconnaissent comme des êtres pensants. Ils s'attendent à ce que l'art parle du réel «de manière symbolique, en utilisant des détournements ${ }^{18}$ ». D'après son expérience, la pédopsychiatre Frédérique Van Leuven en a conclu que «quand on prend la peine de les interroger, ils ont un regard assez lucide sur leur réalité quotidienne ${ }^{19}$ ».

\footnotetext{
16. Ibid., p. 43-44.

17. Entretien avec F. Melquiot réalisé par F. Lhermitte, Journal de la Comédie-Française, $\mathrm{n}^{\circ} 7$, septembre 2003.

I8. J. Pommerat cité par A. Gablin, Le théâtre jeune public : un espace en débat, mémoire de recherche, Institut d'études politiques de Toulouse, 2007, p. 24.

19. F. Van Leuven, «Docteur, je voudrais savoir quelle maladie a ma mère», Enfances \& Psy, $\mathrm{n}^{\circ} 37,2007$, p. 34 .
} 
Évoquer un sujet comme la maladie mentale est en quelque sorte une forme de respect dû à l'intelligence de l'enfant. Les expressions «jeune spectateur» ou «jeune lecteur" ne sont en aucun cas synonymes de "spectateur idiot" ou «lecteur idiot». Les auteurs dramatiques ne se donnent pas de consigne sur les thèmes abordés par leurs pièces car la difficulté réside dans le fait de trouver le bon détournement, selon l'expression de Joël Pommerat, pour évoquer un sujet sensible. La maladie mentale est abordée grâce à des personnages fantastiques, le jeu avec les codes du théâtre (la métathéâtralité, les masques), le rêve éveillé, l'humour... Même si une situation semble triste à première vue, les auteurs donnent une grande importance à l'espoir et à la possibilité d'une guérison... Si l'angélisme n'est pas de mise quand on parle de folie, de maladie mentale ou de troubles de comportement aux enfants, il faut néanmoins garder une certaine légèreté et ne pas oublier que dès que l'on met des mots sur les maux, la maladie mentale devient moins effrayante. Ouais l'a bien compris et explique la maladie avec ses mots à elle : «Un psychopathe, c'est quelqu'un avec le cerveau qui marche à quatre pattes dans sa tête, alors il déraille ${ }^{20}$ \%.

20. F. Melquiot, Blanches, Paris, L’Arche, coll. "Théâtre jeunesse», 20ıо, p. I9. 